\title{
Applications of Magnetized Plasma to Particle Acceleration
}

\author{
Gennady Shvets*†, Jonathan S. Wurtele ${ }^{* *}$ and Min-Sup Hur* \\ ${ }^{*}$ Illinois Institute of Technology, Chicago IL 60302 \\ ${ }^{\dagger}$ Fermi National Accelerator Laboratory, Batavia IL 60510 \\ ${ }^{* *}$ Department of Physics, University of California, Berkeley CA 94720 \\ ${ }^{*}$ Lawrence Berkeley National Laboratory, Berkeley CA 94720
}

\begin{abstract}
Magnetized plasma can be used as an accelerating structure capable of supporting large amplitude longitudinal fields which are externally driven by a high-frequency microwave source. Such structures can be used at very high frequencies (hundreds of gigahertz), placing them in the intermediate region between conventional (metallic) accelerators, and laser-driven plasma accelerators. We review two magnetic field configurations with respect to the direction of the particle beam propagation: (1) parallel magnetic field plus a helical undulator, and (2) perpendicular magnetic field. In the first configuration, plasma exhibits electromagnetically induced transparency (EIT) at the cyclotron frequency if the plasma frequency is equal to the electron cyclotron frequency. The second configuration corresponds to the inverse Cherenkov effect in magnetized plasma. In both cases, the group velocity of the accelerating plasma wave can be made very small, so that the incident electromagnetic wave is strongly compressed, resulting in the high accelerating gradient.
\end{abstract}

\section{INTRODUCTION}

The quest to higher accelerating gradients (and, consequently, shorter and more practical accelerators) inevitably points to higher frequencies of the accelerating field. This is because, according to the dark current trapping criterion, the accelerating gradient $W_{z}<$ $m c \omega / e$, where $\omega$ is the angular frequency, and $e$ and $m$ are the electron charge and mass, respectively. In a conventional accelerating structure higher frequencies also imply smaller feature size which are hard to fabricate, and can be easily damaged by singlepulse Ohmic heating and the resulting cyclic stress [1]. Recent experience of operating high-gradient structures at CLIC and SLAC revealed that, over time, they are damaged by the localized breakdowns in the vicinity of irises. Therefore, it appears unlikely that future metallic accelerators will be operated at a shorter than $1 \mathrm{~cm}$ wavelength.

At the other end of the frequency spectrum are the laser-driven plasma accelerators. Laser wakefield and plasma beatwave accelerators require short-pulse high power laser pulses because plasma wave is driven via the nonlinear ponderomotive force. Typical plasma densities are of order $10^{17}-10^{19} \mathrm{~cm}^{-3}$, and plasma wavelength $10 \mu m<\lambda_{p}<$ $100 \mu \mathrm{m}$. Low laser efficiency and repetition rate are some of the technical challenges on the path to developing a practical laser-plasma accelerator.

In this paper we look at the previously neglected regime of intermediate frequencies (hundreds of $\mathrm{GHz}$ ) and plasma densities (of order $10^{14}-10^{15} \mathrm{~cm}^{-3}$ ) where high power microwaves can be directly converted into plasma waves. It is also possible to find the

CP647, Advanced Accelerator Concepts: Tenth Workshop, edited by C. E. Clayton and P. Muggli (C) 2002 American Institute of Physics 0-7354-0102-0/02/\$19.00

681 
appropriate parameter regime which corresponds to the slow group velocity $v_{g} \ll c$ of the resulting plasma waves. The incident radio-frequency pulse is compressed in the plasma by the factor $c / v_{g} \gg 1$, and converted into a predominantly longitudinal wave capable of accelerating charged particles to high energies. Therefore, plasma can serve as both the power compressor, and the accelerator.

Two magnetic field configurations are considered. The first one, described in Sec. 1, is the combination of the axial and helical magnetic fields. It is inspired by the undulatorinduced transparency of magnetized plasma at the cyclotron frequency [2]. The transparency condition requires that $\omega_{p}=\Omega_{0}$, where $\omega_{p}=\left(4 \pi e^{2} n / m\right)^{1 / 2}$ is the plasma frequency, and $\Omega_{0}=e B_{0} / m c$ is the cyclotron frequency, with $B_{0}$ the amplitude of the axial magnetic field.

The second magnetic configuration described in Sec. 2 only uses magnetic field which is perpendicular to the acceleration direction. Injecting an intense electromagnetic wave into the magnetized plasma and using it to accelerate particles is an inverse process to the Cherenkov radiation in magnetized plasma described by Yoshii et. al. [3]. For both magnetic field configurations, electromagnetic wave is injected at the plasma frequency $\omega=\omega_{p}$. Magnetic field of arbitrary strength can be used in an Inverse Cherenkov Accelerator in Magnetized Plasma (ICAMP).

\section{UNDULATOR INDUCED TRANSPARENCY OF THE MAGNETIZED PLASMA}

It is well known that a uniformly magnetized plasma $\left(\vec{B}_{0}=\vec{e}_{z} B_{0}\right)$ is strongly heated by the right-hand polarized (RHP) electromagnetic wave with the frequency $\omega_{1}=\Omega_{0}$. As the result of the strong interaction between the electromagnetic wave and the plasma near the electron cyclotron resonance, a stopband develops for $\Omega_{0}<\omega_{1}<\Omega_{R H}$, where $\Omega_{R H}=\sqrt{\Omega_{0}^{2} / 4+\omega_{p}^{2}}+\Omega_{0} / 2$ is the right-hand cutoff frequency. The corresponding dispersion relation $\omega$ v. s. $k$ is shown in Fig. 1.

The purpose of this Section is to demonstrate that the electron cyclotron heating ECR can be prevented, and plasma made transparent at the cyclotron frequency by exploiting the effect of electromagnetically induced transparency (EIT). Several applications which are the focus of this paper are enabled by EIT: microwave-plasma acceleration of electrons and ions.

In the classical plasma EIT can be caused by either a strong EM wave [4], or a magnetostatic helical undulator [2]. The latter option requires that $\Omega_{0}=\omega_{p}$. Using an undulator is more practical because no strong electromagnetic pump is required, and the entire energy of the incident wave can be converted into a plasma wave and used for particle acceleration. Below we concentrate on the undulator induced plasma transparency.

The mechanism of the undulator induced transparency (UIT) is the destructive interference between the electric force of the probe $\left(-e \vec{E}_{1 \perp}\right)$ and the magnetic force of the undulator $\left(-e v_{z} \vec{e}_{z} \times \vec{B}_{u} / c\right)$ exerted on the collective electron plasma oscillation along the magnetic field. The total force at the cyclotron frequency experienced by a plasma 


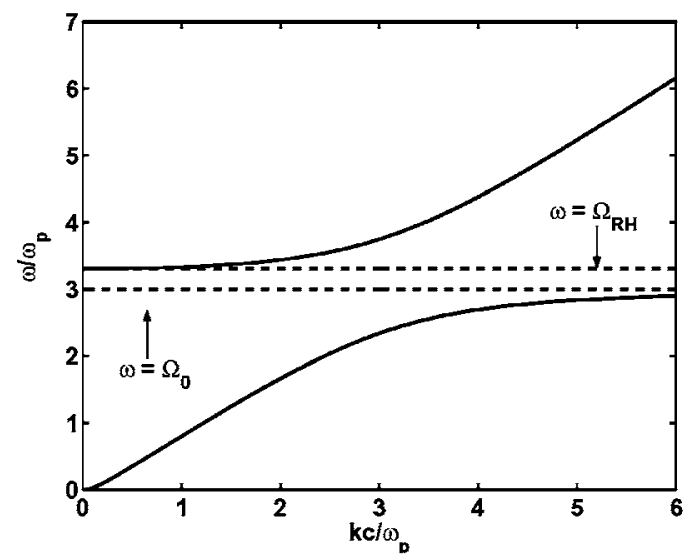

FIGURE 1. Dispersion relation for a right-hand polarized wave propagating along magnetic field. Forbidden gap exists between cyclotron frequency $\Omega_{0}$ and the right-hand cutoff frequency $\Omega_{R H}=\Omega_{0} / 2+$ $\sqrt{\Omega_{0}^{2} / 4+\omega_{p}^{2}}$.

electron is given by $\vec{F}_{\text {tot }} \approx-e\left(\vec{E}_{1 \perp}+\dot{\zeta}_{z} \vec{e}_{z} \times \vec{B}_{u}\right)$, where $\zeta_{z}$ is the electron displacement in the plasma wave. With only the probe wave present, the resonant $\vec{E}_{1 \perp}$ generates a large plasma current which causes the wave to be absorbed by the plasma. With both the pump and probe present, the net force can vanish $\left(\vec{F}_{\text {tot }} \sim 0\right)$. Consequently, the plasma current at the cyclotron frequency is small (or even vanishing), and the probe propagates almost as if in vacuum. Single-particle numerical simulation below demonstrates that this situation is naturally achieved in a collisionless plasma.

Consider an externally magnetized plasma with $\vec{B}=B_{0} \vec{e}_{z}$ and density $n_{0}$. We assume a right-hand polarized EM probe propagating along $z$ - direction, with its electric and magnetic fields given by $2 e \vec{E}_{1 \perp} / m c \omega_{1}=a_{\text {probe }} \vec{e}_{+} \exp \left(i \vec{\theta}_{1}\right)+c . c$., and $\vec{B}_{1 \perp}=$ $\left(c \vec{k}_{1} / \omega_{1}\right) \times \vec{E}_{1 \perp}$, where $\vec{e}_{ \pm}=\vec{e}_{x} \pm i \vec{e}_{y}$, and $\bar{\theta}_{1}=k_{1} z-\omega_{1} t$. The undulator field is given by $2 e \vec{B}_{u} / m c^{2} k_{0}=a_{0} \vec{e}_{+} \exp \left(i \bar{\theta}_{0}\right)+c . c$., where $\bar{\theta}_{0}=k_{0} z$. Note that the helicity of the undulator is determined by whether $a_{0}$ is real (left helicity) or imaginary (right helicity).

The non-relativistic equation of motion of a plasma electron in the combined fields is given by

$$
\frac{d^{2} \vec{x}}{d t^{2}}+\Omega_{0} \vec{v} \times \vec{e}_{z}+\omega_{p}^{2} \zeta_{z} \vec{e}_{z}=-\frac{e}{m} \sum_{m=0,1} \vec{E}_{m \perp}+\frac{\vec{v} \times \vec{B}_{\perp m}}{c}
$$

where $\vec{x} \equiv\left(z_{0}+\zeta_{z}\right) \vec{e}_{z}+\vec{x}_{1}$ and $\vec{v}=d \vec{x} / d t \equiv c \vec{\beta}$ are the particle position and velocity. The initial conditions are $\vec{v}=0$ and $\vec{x}=z_{0} \vec{e}_{z}$. The third term on the LHS of Eq. (1) is the restoring force of the ions.

Equation (1) was integrated for the resonant case of $\omega_{p}=\Omega_{0}$. Undulator parameters are $a_{0}=0.1$ and $k_{0}=2 \Omega_{0} / c$, and the probe is switched on according to $a_{\text {probe }}=0.5 a_{1}\left(1+\tanh \left[\left(\Omega_{0} t-320\right) / 60\right]\right)$, where $a_{1}=0.01$. Suppression of the electron response at the cyclotron frequency is apparent from Fig. 2(a), where $\beta_{x}$ is plotted 

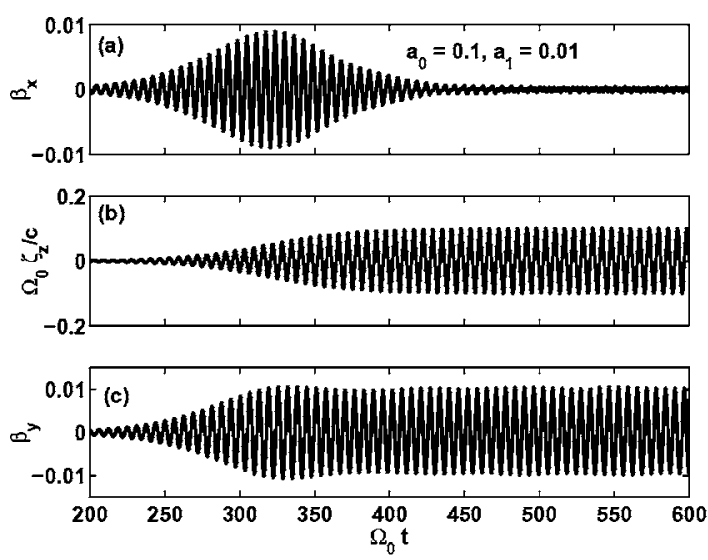

FIGURE 2. Numerical simulation of single particle motion (Eq. 1) in the combined field of an adiabatically turned on EM probe (with $a_{1}=0.01, \omega_{1}=\Omega_{0}, k_{1}=\Omega_{0} / c$ ) and a static helical undulator (with $a_{0}=0.1$ and $k_{0}=2 \Omega_{0} / c$ ). Plasma density corresponds to $\omega_{p}=\Omega_{0}$. (a) Transverse velocity $\beta_{x}$, (b) longitudinal displacement $\Omega_{0} \zeta_{z} / c$ during and after the turn-on of the probe, and (c) transverse velocity $\beta_{y}$.

as a function of time. Without the undulator $\beta_{x}$ would have grown without bound because it is resonantly driven by the probe. The force due to the electric field of the probe is cancelled by the $\left(\dot{\zeta}_{z} / c\right) \vec{e}_{z} \times \vec{B}_{0 \perp}$ force which is exerted on a longitudinal plasma wave by the helical magnetic field of the undulator. The normalized amplitude of the plasma wave $\Omega_{0} \zeta_{z} / c$ is plotted in Fig. 2(b). The plasma wave (which is $10 \%$ of the wavebreaking limit for this example) can be used for acceleration of relativistic electrons because its phase velocity is $v_{p h}=\left(\omega_{1}-\omega_{0}\right) /\left(k_{1}-k_{0}\right) \approx-c$.

Since the probe is right hand polarized, it is tempting to assume that the electron gyration will also possess the same handedness. Handedness is defined by expressing $\vec{\beta}=\left(\beta_{+} \vec{e}_{+}+\beta_{-} \vec{e}_{-}\right) / 2$, where $\beta_{+}=\beta_{-}^{*}=\beta_{x}-i \beta_{y}$. If $\beta_{+} \propto \exp (-i \omega t)$, then the electron motion is right-handed. This assumption turns out to be wrong: electron motion is a superposition of the right and left-handed rotations. In fact, if $\beta_{+}=$ $D \exp (-i \omega t)-D \exp (i \omega t)$ (where $D$ is an arbitrary real constant), then electron oscillates in $y$-direction. This situation is captured by Figs. 2(a,c) which show that the $x-$ component of the electron velocity vanishes while the $y$-component does not. It will be shown that the relative phase and amplitudes of the the right- and left-handed rotations depends on the electron position.

Below we show how this polarization rotation can be explained by the interaction between the plasma wave oscillation and the undulator field. The steady-state values of $\beta_{+}$and $\zeta_{z}$ can be analytically obtained by a straightforward linearization of Eq. (1) in the weak probe limit of $a_{1} \ll a_{0}$ as was shown in Ref. [2]:

$$
\dot{\beta}_{+}+i \Omega_{0} \beta_{+}=-\omega_{0} a_{0} e^{i \theta_{0}}\left(1+i k_{0} \zeta_{z}-k_{0} \dot{\zeta}_{z} / \omega_{0}\right)-\omega_{1} a_{1} e^{i \theta_{1}}
$$


is for the transverse motion, and

$$
\ddot{\zeta}_{z}+\omega_{p}^{2} \zeta_{z}=-\frac{c^{2}}{2}\left(k_{0} a_{0} \beta_{-} e^{i \theta_{0}}+k_{1} a_{1} \beta_{-} e^{i \theta_{1}}-i k_{0}^{2} \zeta_{z} \beta_{-} a_{0} e^{i \theta_{0}}\right)+c . c .
$$

for the longitudinal motion. We have introduced $\theta_{1}=k_{1} z_{0}-\omega_{1} t, \theta_{0}=k_{0} z_{0}$, and assumed that $k_{0,1} \zeta_{z}<1$.

Further, the steady-state solution of Eq. (3) is assumed to be $\zeta_{z}=0.5 \tilde{\zeta} \exp i\left(\Delta k z-\omega_{1} t\right)+c . c$. , where $\Delta k=k_{1}-k_{0}$, and substituted into Eq. (2). Retaining terms with $\exp \pm i \omega_{1} t$ dependence results in

$$
\beta_{+}=-\frac{i \omega_{1}}{\omega_{1}-\Omega_{0}}\left(a_{1}+\frac{i k_{0} \tilde{\zeta}}{2} a_{0}\right) e^{i \theta_{1}}+\frac{\omega_{1} a_{0}}{\omega_{1}+\Omega_{0}}\left(\frac{k_{0} \tilde{\zeta}}{2}\right) e^{i\left(2 \theta_{0}-\theta_{1}\right)} .
$$

The second term in the RHS of the Eq. (4) is the left-hand polarized component of the electron velocity omitted in Ref. [2]. It appears because the magnetic force of the undulator acting on a plasma wave has two components: the resonant right hand polarized and the non-resonant left hand polarized ones. From Eq. (4) the steady-state amplitude of the plasma wave is found for $\omega_{1}=\Omega_{0}:|\tilde{\zeta}|=2 a_{1} / a_{0} k_{0}$. Transverse velocity is found by inserting $\beta_{+}$and $\beta_{-}$into Eq. (3):

$$
\left(\Omega_{0}^{2}-\omega_{1}^{2}\right) \tilde{\zeta}=i c^{2}\left[\frac{k_{0} a_{0}^{*} \omega_{1}}{\omega_{1}-\Omega_{0}}\left(a_{1}+i k_{0} \tilde{\zeta} a_{0} / 2\right)+i \frac{k_{0}^{2} \tilde{\zeta} \omega_{1}}{\omega_{1}+\Omega_{0}}\left|a_{0}\right|^{2}\right],
$$

where we have explicitly used $\omega_{p}=\Omega_{0}$ in the LHS. Solving Eq. (5) for $\tilde{\zeta}$ and substituting into Eq. (4) yields the steady-state perpendicular velocity:

$$
\beta_{+s} \approx i a_{1} \frac{\omega_{1} \delta \Omega-\Omega_{R}^{2} / 2}{\delta \Omega^{2}-\Omega_{R}^{2}} e^{i \theta_{1}}+i a_{1}^{*} \frac{ \pm \Omega_{R}^{2} / 2}{\delta \Omega^{2}-\Omega_{R}^{2}} e^{i\left(2 \theta_{0}-\theta_{1}\right)},
$$

where $\delta \Omega=\omega_{1}-\Omega_{0}$ is the detuning, $\Omega_{R}=c k_{0} a_{0} / 2$ is the effective Rabi frequency, and the plus (minus) sign corresponds to the left (right) undulator helicity. The dispersion relation for the probe becomes

$$
\omega_{1}^{2}=c^{2} k_{1}^{2}-\omega_{p}^{2} \frac{\omega_{1}(\delta \Omega)-\Omega_{R}^{2} / 2}{\Omega_{R}^{2}-(\delta \Omega)^{2}},
$$

indicating that the complete transparency (vacuum-like propagation) is achieved for $\delta \Omega \approx \Omega_{R}^{2} / 2 \Omega_{0} \ll \Omega_{R}$. Another implication of the Eq. (7) is that the propagation bandwidth $\left|\delta \Omega \ll \Omega_{R}\right|$ is much larger than that of the vacuum-like propagation (where refraction index is of order unity).

The EIT dispersion relation is plotted in Fig. (3) for the same plasma parameters as in Fig. 2 and an undulator with $\Omega_{R}=0.5 \omega_{p}$. The flat band between the $\Omega_{0} \pm \Omega_{R}$ resonant frequencies is the classical analog of the "slow light" in atomic systems. The corresponding group velocity $v_{g}=\partial \omega_{1} / \partial k_{1} \approx 2 c \Omega_{R}^{2} / \omega_{p}^{2}$ can also be made very small. Detailed numerical studies of the dispersion relation are described in Ref. [5]. 


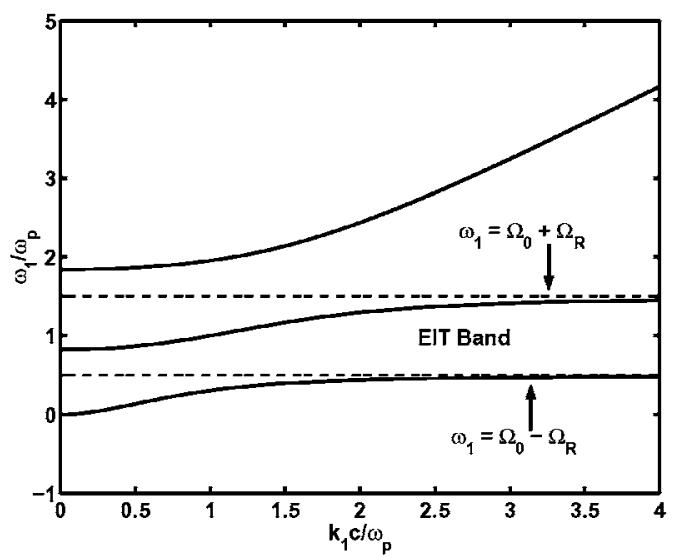

FIGURE 3. EIT dispersion curve, $\Omega_{0}=\omega_{p}$ and $\Omega_{R} / \omega_{p}=1 / 2$. Flat band above $\Omega_{0}-\Omega_{R}$ up to $\Omega_{0}+\Omega_{R}$ labeled "EIT Band" corresponds to "slow light" in the presence of an undulator.

Slowing down of electromagnetic waves in the EIT plasma can be understood by considering the entrance of a probe beam of duration $L_{0}$ into the plasma. In the plasma, the "slow light" of length $L_{f}$ consists of the transversely polarized field of the probe $\left|\vec{E}_{1}\right|=\left|\vec{B}_{1}\right|=a_{1} m c \omega_{1} / e$ and the longitudinal electric field of the plasma wave $E_{Z}=$ $4 \pi e n_{0}\left(2 a_{1} / k_{0} a_{0}\right)$. As the pulse enters the plasma, it loses photons to the pump at the same rate as new plasmons are created (according to the Manley-Rowe relations). The classical photon density of a field with frequency $\omega$ is proportional to the action density $\propto U / \omega$, where $U$ is the energy density. From this one can calculate that

$$
\frac{U_{\text {plas }} / \omega_{p}}{U_{\text {phot }} / \omega_{1}}=\frac{\Omega_{0}}{\omega_{p}} \frac{E_{z}^{2}}{2 E_{1}^{2}}=\frac{\omega_{p}^{2}}{2 \Omega_{R}^{2}} \gg 1 \text { for } \Omega_{R} \ll \omega_{p}
$$

Thus, most of the original pulse photons are transferred to the plasma wave. Since $c k_{1} \approx \omega_{1}$, the photon energy does not change. Therefore, the loss of photons is due to the spatial shortening of the pulse from $L_{0}$ to $L_{f}=L_{0} \times\left(2 \Omega_{R}^{2} / \omega_{p}^{2}\right)$. Because temporal pulse duration does not change, we recover the previously calculated $v_{g} / c=2 \Omega_{R}^{2} / \omega_{p}^{2}$. In the case of a static undulator all the energy is transfered to the plasma and compressed by a factor $v_{g} / c$, resulting in a dramatic increase of the energy density.

One interesting application of EIT in magnetized plasma is ion acceleration. EIT enables a short-pulse ion accelerator consisting of a "slow light" pulse in the plasma with approximately equal group and phase velocities. Acceleration is accomplished by the longitudinal electric field of the plasma wave. Phase velocity $v_{\mathrm{ph}}=\omega_{p} /\left(\left|k_{0}\right|+k_{1}\right)$ can be easily controlled by slightly changing the wave frequency or magnetic field strength. 


\section{INVERSE CHERENKOV ACCELERATOR IN MAGNETIZED PLASMA (ICAMP)}

In this Section we propose another scheme of a microwave-driven plasma accelerator. As in the case of EIT, the goal is to create an accelerating medium (magnetized plasma) with the following electromagnetic properties with respect to the accelerating electromagnetic wave propagating in $x$ - direction. (i) Low group velocity: $v_{g}=\partial \omega / \partial k \ll c$. This enables significant pulse compression, making the energy density inside the plasma much higher than in the transporting waveguide. (ii) Mostly longitudinal polarization: $E_{x} \gg E_{z}, E_{y}$. Such a wave is capable of accelerating particles, and has a high shunt impedance. (iii) Luminous phase velocity: $v_{\mathrm{ph}}=\omega / k=c$ is equal to the speed of light. This property is essential for accelerating particles to relativistic energies. It is also important for high coupling efficiency from vacuum to plasma (impedance matching).

It turns out that such a configuration was already considered by Yoshii et. al. [3] for generating high power radiation. Cherenkov wakes with luminous phase velocity in $x$ - direction are excited by a short particle or laser beam in the plasma immersed in uniform magnetic field $\vec{B}=B_{u} \vec{e}_{z}$. It was clearly demonstrated [6] using 2-D particle-incell simulations that a short particle bunch moving perpendicularly to the magnetic field excites a slowly propagating electromagnetic wave in magnetized plasma. Of course, any radiation source which uses the kinetic energy of a charged particle can be operated "in reverse" and used as an accelerator. In an accelerator the externally supplied energy of the electromagnetic field is transferred to the charged particle bunch. To our knowledge, the idea of injecting microwaves into the magnetized plasma with the purpose of particle acceleration via the inverse Cherenkov mechanism is put forward for the first time.

PIC simulations using the one-dimensional version of the Virtual Laser Plasma Laboratory (VLPL) code developed by A. Pukhov were carried out. We modeled the coupling of a planar electromagnetic wave incident from vacuum region into the plasma. Wave frequency $\omega=\omega_{p}$ matches the plasma frequency, and its amplitude $e E_{y} / m c \omega=0.01$. Plasma is magnetized in $z$ - direction, with $\vec{B}=B_{u} \vec{e}_{z}$, where $e B_{u} / m c \omega_{p}=0.3$. The microwave pulse duration is $T=100 \lambda_{p} / c$, where $\lambda_{p}=2 \pi c / \omega$. Plasma occupies the region $110<X / \lambda_{p}<120$.

Three snapshots are shown in Fig. 4. At $t=0$ [Fig. 4(a)] the electromagnetic pulse approaches the plasma from the left. By $t=100 \lambda_{p} / c$ [Fig. 4(b)] most of the microwave pulse had entered the plasma and converted into the (primarily longitudinal) Cherenkov wake. The compression factor is of order 10 . The ratio between the longitudinal electric field in the plasma $E_{x}$ in the plasma and the transverse field in vacuum (and in the plasma) is about 3.5. Therefore, ICAMP has a high shunt impedance: most of the field energy is participating in particle acceleration. At a later time $t=200 \lambda_{p} / c$ [Fig. 4 (c)] almost all electromagnetic energy had moved out of the plasma. Very little absorption or reflection is observed.

We have also verified that the phase velocity of the Cherenkov wake is exactly equal to the speed of light by measuring its wavelength in the plasma. We found that $\lambda=\lambda_{p}$, so that $v_{p h}=\lambda \omega / 2 \pi=c$. The exact matching of the refraction index between the magnetized plasma and vacuum is responsible for the high coupling efficiency from vacuum into the plasma. 

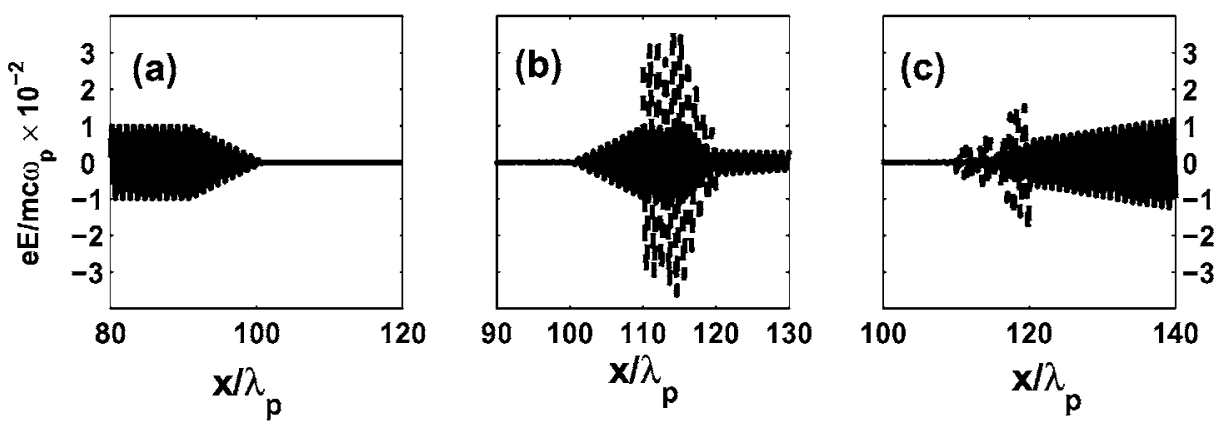

FIGURE 4. Cherenkov wakefield accelerator in magnetized plasma. Magnetic field $\vec{B}=\vec{e}_{z} B_{u}$, $e B_{u} / m c \omega_{p}=0.3$. Plasma in region $110<x / \lambda_{p}<120$. Electromagnetic wave with $\omega=\omega_{p}$ and $e E_{y} / m c \omega=0.01$ is incident from vacuum. Transverse $\left(E_{y}\right)$ and accelerating $\left(E_{x}\right)$ electric fields at different times: (a) $t=0$, (b) $t=100 \lambda_{p} / c$, and (c) $t=200 \lambda_{p} / c$. Solid line: $E_{y}$, dashed line: $E_{x}$.

Theoretically, phase velocity is expected to be equal to the speed of light for $\omega=\omega_{p}$. This can be seen from the well known dispersion relation of the extraordinary (XO) EM wave propagating perpendicularly to the magnetic field:

$$
\frac{c^{2} k^{2}}{\omega^{2}}=1-\frac{\omega_{p}^{2}}{\omega^{2}} \frac{\omega^{2}-\omega_{p}^{2}}{\omega^{2}-\omega_{H}^{2}}
$$

where $\omega_{H}^{2}=\omega_{p}^{2}+\Omega_{u}^{2}$ and $\Omega_{u}=e B_{\mathrm{u}} / m c$. Therefore, $k c=\omega$ for $\omega=\omega_{p}$. The projected group velocity is $v_{g} / c=\Omega_{u}^{2} / \omega_{H}^{2}$. For the presented simulation parameters $v_{g} / c \approx 0.1$ in good agreement with the simulation results.

It is instructive to translate the simulation results presented in Fig. (4) into physical numbers by assuming that $B_{u}=1$ Tesla, $\omega / 2 \pi=100 \mathrm{GHz}$ (or $\lambda=3 \mathrm{~mm}$ ), and $n_{p}=$ $10^{14} \mathrm{~cm}^{-3}$. Assuming that the plasma area is $\lambda^{2}=0.1 \mathrm{~cm}^{2}$, the incident microwave power is about $3 \mathrm{MW}$. The wavebreaking field is about $1 \mathrm{GV} / \mathrm{m}$, and the simulated accelerating wake is $36 \mathrm{MV} / \mathrm{m}$. Increasing the incident microwave power to $300 \mathrm{MW}$ should increase the accelerating gradient to about $360 \mathrm{MV} / \mathrm{m}$.

Viability of the ICAMP for high energy physics applications depends on whether electrons can gain energy from the accelerating field faster than losing it in the form of a synchrotron radiation. For ultra-relativistic electrons the radiated power is given by $P=(2 / 3) e^{2} c \gamma^{4} / \rho^{2}$, where $\rho=\gamma c / \Omega_{u}$ is the radius of curvature. For $\gamma=10^{5}$ and $B_{u}=1 \mathrm{~T}$ we find that $\rho=160 \mathrm{~m}$. Therefore, in a $L=1 \mathrm{~m}$ long accelerating section the beam is transversely displaced by $\delta y=L^{2} / 2 \rho \approx 30 \mu \mathrm{m}$. Synchrotron loss per unit length is given by

$$
\frac{d P}{d z}=\frac{2}{3} \gamma^{2} m c^{2}\left(\frac{\Omega_{u}^{2} r_{e}}{c^{2}}\right)=3 \mathrm{MV} / \mathrm{m}\left(10^{-10} \gamma^{2} B_{u}^{2}\right)
$$


where $r_{e}=e^{2} / m c^{2}$ is the classical electron radius, and $B_{u}$ is measured in Tesla. Thus, for $B_{u}=1 \mathrm{~T}$ and $\gamma=10^{5}$ synchrotron losses are about $1 \%$ of the energy gain due to acceleration. This constraint on the performance of the inverse Cherenkov accelerator becomes significant beyond $100 \mathrm{GeV}$ unless a denser plasma (or lower magnetic field) is used.

\section{CONCLUSIONS}

Both UTT-based and inverse Cherenkov accelerators aim at solving the same problem: how to efficiently couple high-frequency microwave radiation into the plasma, thereby transforming it into a longitudinal wave capable of accelerating high energy electrons. Both schemes share similar features: (1) luminous waves with $\omega=k c$ are possible; (2) there are no reflections upon coupling from vacuum into the plasma; (3) group velocities scale as $\Omega_{u}^{2} / \omega_{p}^{2}$; (4) electric field in the plasma is primarily longitudinal; (5) very large energy density compression is achievable. Although the ICAMP appears conceptually simpler because no guide field is required, having a strong guiding field may actually be advantageous for ensuring plasma homogeneity along the propagation direction. Additional numerical simulations in 2 and 3-D are required to assess the relative merits of the two schemes.

\section{ACKNOWLEDGMENTS}

We gratefully acknowledge the contribution of Dr. A. Pukhov for simulating the inverse Cherenkov accelerator during this workshop. This work was supported by the US DOE Division of High-Energy and Nuclear Physics and (for G. S.) by the Presidential Early Career Award for Scientists and Engineers (PECASE), the Illinois Board of Higher Education, and the the Illinois Department of Commerce and Community Affairs.

\section{REFERENCES}

1. D. H. Whittum, in Advanced Accelerator Concepts, edited by W. Lawson, C. Bellamy, and D. F. Brosius, AIP Conf. Proc. No. 472 (AIP, New York, 1999), pp. 72-85.

2. G. Shvets and J. S. Wurtele, Phys. Rev. Lett, in press (2002).

3. J. Yoshii et. al., Phys. Rev. Lett. 79, 4194 (1997).

4. A. G. Litvak and M. D. Tokman, Phys. Rev. Lett. 88, 095003 (2002).

5. M. S. Hur, J. S. Wurtele, and G. Shvets, "Magnetically Induced Transparency and Its Application as an Accelerator", these proceedings.

6. N. Spence et. al., Phys. Plasmas 8, 4995 (2001). 